\title{
Metallic Thermal Conductivity in a Magnetic Insulator
}

\section{Featured paper:}

1. "Highly Mobile Gapless Excitations in a Two-Dimensional Candidate Quantum Spin Liquid"

Authors: M. Yamashita, N. Nakata, Y. Senshu, M. Nagata, H. M. Yamamoto, R. Kato, T. Shibauchi and Y. Matsuda, Science 328, 1246 (2010)

\section{Recommended with a commentary by Ashvin Vishwanath, UC Berkeley}

Typically, one expects excellent thermal conductors to also be electrically conducting. At room temperature there are a few exceptions to this rule, notably diamond which is an insulator where phonons lead to large thermal conductivity. However, at very low temperatures it was long believed that only metals could conduct heat well. More precisely, the ratio of thermal conductivity $(\kappa)$ to temperature vanishes in the low temperature limit in all other known phases, in two and three dimensions. For example, phonons in a bulk crystal have a low temperature thermal conductivity that varies as $\kappa \sim T^{3}$. In metals however this ratio approaches a constant, which is related by the Wiedemann-Franz law to the electrical $(\sigma)$ conductivity, via universal constants.

$$
\lim _{T \rightarrow 0} \kappa / T=\sigma\left(\frac{\pi^{2} k_{B}^{2}}{3 e^{2}}\right)
$$

Essentially, this large thermal conductivity arises from the large number of low energy excitations in a metal, in the vicinity of the Fermi surface.

The featured reference reports on a experiments that shakes this conventional wisdom. Thermal conductivity of an organic material EtMe $\mathrm{Sb}_{3}\left[\mathrm{Pd}(\mathrm{mit})_{2}\right]_{2}$ (henceforth called dmit131) was measured at temperatures as low as 100 milliKelvin. Despite being an electrical insulator, a 'metallic' thermal conductivity was found, with a finite value for the ratio $\kappa / T$. This is the intercept of the pink curve depicted in Figure 1. Moreover, this residual thermal conductivity is extremely large - for example it is comparable to that of brass at low temperatures. Another way to quote the size is in terms of the thermal conductivity per layer, given this is a layered material. Just like with electrical conductivity, there is a universal quantum for the thermal conductivity ratio $\kappa / T$ in two dimensions. The measured value is a factor of $\sim 10^{3}$ times this quantum. This would be the $k_{F} l$ value for a metal, the 


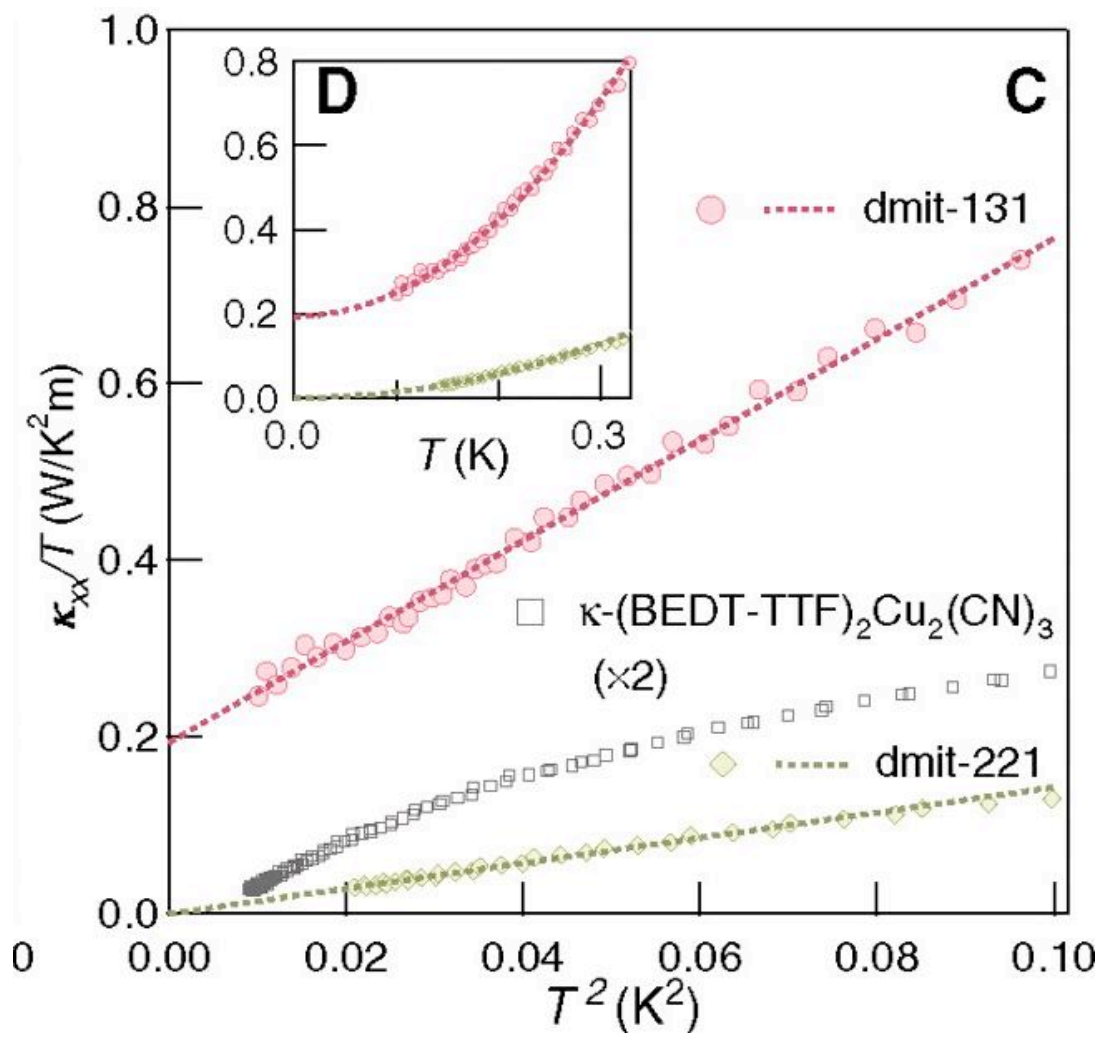

M Yamashita et al. Science 2010;328:1246-1248

FIG. 1: Low temperature thermal conductivity of dmit-131 (pink curve) from the featured reference. The nonzero intersept is the finite value of $\kappa / T$ in the $T \rightarrow 0$ limit. The other curves, with vanishing intersept, refer to conventional insulating phases in sister compounds.

product of Fermi wavevector and scattering length. The big value of the measured signal indicates this is a robust experimental result, largely insensitive to precise fitting of the phonon component of the thermal conductivity at higher temperatures.

Given these are electrical insulators, mobile electrons are unlikely to account for the thermal conductivity. The authors suggest that the spin degrees of freedom are responsible, if they form a quantum spin liquid state. Indeed, these insulators show clear signature of local spin 1/2 moments, arranged on the sites of a triangular lattice. Despite a fairly large exchange constant, $J \sim 250 K$ as inferred from the Curie-Weiss susceptibility, no magnetic order or spin freezing was observed down to the lowest subKelvin temperatures measured[1]. On the theory side[2], it has been argued that a particular type of spin liquid, the spinon 
Fermi surface state, is energetically favorable for the spin Hamiltonians likely to occur in these materials. These states can be caricatured as retaining the spin correlations of a Fermi liquid, but which are insulating. An experimental observation that is invoked is that under moderate pressure, it is known that the dmit-131 material undergoes a transition into a conventional metallic state. The spinon Fermi surface state can be thought of as a natural intermediate phase, halfway between a conventional magnetic insulator and a metal. At a crude level, many experimental facts resemble that of a metal whose charge has been stripped away. This includes the thermal conductivity, but also specific heat and magnetic susceptibility in this[1] and a related organic material $\kappa(\mathrm{ET})_{2} \mathrm{Cu}_{2}(\mathrm{CN})_{3}$ [3], that resemble those of a charge neutral fermi liquid.

A more sophisticated theoretical treatment of this spin liquid state including interactions between the spin carrying fermions, predicts deviations at low temperatures, notably a thermal conductivity enhancement as temperature is lowered. This has not been observed experimentally as yet. Clearly much more work needs to be done to meaningfully compare theoretical results with experiments. At a more basic level, it would be nice to see direct experimental evidence for the spins being the dominant low temperature excitations. Irrespective of the theoretical scenario, explaining the remarkable phenomenon of metallic heat conduction in these electrical insulators promises to lead to interesting developments.

[1] T. Itou et al., Phys. Rev. B 77, 104413 (2008)

[2] O. Motrunich Phys. Rev. B 72, 045105 (2005). S. S. Lee \& P. A. Lee, Phys. Rev. Lett. 95, 036403 (2005).

[3] Y. Shimizu et al., Phys. Rev. Lett. 91, 107001 (2003). 